\title{
AGILE detection of a rapid $\gamma$-ray flare from the blazar PKS 1510-089 during the GASP-WEBT monitoring ${ }^{\star}$
}

F. D’Ammando ${ }^{1,2}$, G. Pucella ${ }^{1}$, C. M. Raiteri ${ }^{3}$, M. Villata ${ }^{3}$, V. Vittorini ${ }^{1,4}$, S. Vercellone ${ }^{5}$, I. Donnarumma ${ }^{1}$, F. Longo ${ }^{6}$, M. Tavani ${ }^{1,2}$, A. Argan ${ }^{1}$, G. Barbiellini' ${ }^{6}$, F. Boffelli ${ }^{7,8}$, A. Bulgarelli ${ }^{9}$, P. Caraveo ${ }^{10}$, P. W. Cattaneo ${ }^{7}$, A. W. Chen ${ }^{4,10}$, V. Cocco ${ }^{1}$, E. Costa ${ }^{1}$, E. Del Monte ${ }^{1}$, G. De Paris ${ }^{1}$, G. Di Cocco ${ }^{9}$, Y. Evangelista ${ }^{1}$, M. Feroci ${ }^{1}$, A. Ferrari ${ }^{11}$, M. Fiorini ${ }^{10}$, T. Froysland ${ }^{2,4}$, F. Fuschino ${ }^{9}$, M. Galli ${ }^{12}$, F. Gianotti ${ }^{9}$, A. Giuliani ${ }^{10}$, C. Labanti ${ }^{9}$, I. Lapshov ${ }^{1}$, F. Lazzarotto ${ }^{1}$, P. Lipari ${ }^{13}$, M. Marisaldi ${ }^{9}$, S. Mereghetti ${ }^{10}$, A. Morselli ${ }^{14}$, L. Pacciani ${ }^{1}$, A. Pellizzoni ${ }^{15}$, F. Perotti ${ }^{10}$, G. Piano ${ }^{1,2}$, P. Picozza ${ }^{14}$, M. Pilia ${ }^{15}$, M. Prest ${ }^{16}$, M. Rapisarda ${ }^{17}$, A. Rappoldi ${ }^{7}$, S. Sabatini ${ }^{1,2}$, P. Soffitta ${ }^{1}$, M. Trifoglio ${ }^{9}$, A. Trois ${ }^{1}$, E. Vallazza ${ }^{6}$, A. Zambra ${ }^{1}$, D. Zanello ${ }^{13}$, I. Agudo ${ }^{18}$, M. F. Aller ${ }^{19}$, H. D. Aller ${ }^{19}$, A. A. Arkharov ${ }^{20}$, U. Bach ${ }^{21}$, E. Benitez ${ }^{22}$, A. Berdyugin ${ }^{23}$, D. A. Blinov ${ }^{24}$, C. S. Buemi ${ }^{25}$, W. P. Chen ${ }^{26,27}$, A. Di Paola ${ }^{28}$, G. Di Rico ${ }^{29}$, D. Dultzin ${ }^{22}$, L. Fuhrmann ${ }^{21}$, J. L. Gómez ${ }^{18}$, M. A. Gurwell ${ }^{30}$, S. G. Jorstad ${ }^{31}$, J. Heidt ${ }^{32}$, D. Hiriart ${ }^{33}$, H. Y. Hsiao ${ }^{27}$, G. Kimeridze ${ }^{34}$, T. S. Konstantinova ${ }^{24}$, E. N. Kopatskaya ${ }^{24}$, E. Koptelova2627,

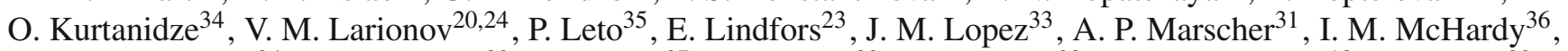

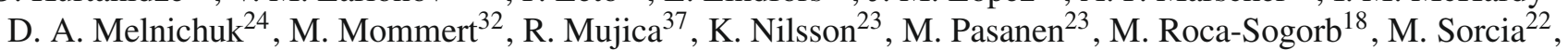
L. O. Takalo $^{23}$, B. Taylor ${ }^{38}$, C. Trigilio ${ }^{25}$, I. S. Troitsky ${ }^{24}$, G. Umana ${ }^{25}$, L. A. Antonelli ${ }^{39}$, S. Colafrancesco ${ }^{39}$, S. Cutini ${ }^{39}$, D. Gasparrini ${ }^{39}$, C. Pittori ${ }^{39}$, B. Preger ${ }^{39}$, P. Santolamazza ${ }^{39}$, F. Verrecchia ${ }^{39}$, P. Giommi ${ }^{39}$, and L. Salotti ${ }^{40}$

(Affiliations can be found after the references)

Received 23 May 2009 / Accepted 15 September 2009

\section{ABSTRACT}

\begin{abstract}
Aims. We report the detection by the AGILE satellite of a rapid $\gamma$-ray flare from the source 1AGL J1511-0908, associated with the powerful $\gamma$-ray quasar PKS 1510-089, during a pointing centered on the Galactic center region from 1 March to 30 March 2008. This source has been continuosly monitored in the radio-to-optical bands by the GLAST-AGILE Support Program (GASP) of the Whole Earth Blazar Telescope (WEBT). Moreover, the $\gamma$-ray flaring episode triggered three ToO observations by the Swift satellite in three consecutive days, starting from 20 March 2008 . The quasisimultaneous radio-to-optical, UV, X-ray and $\gamma$-ray coverage allows us to make a detailed study of the multifrequency time evolution, the spectral energy distribution of this source, and its theoretical interpretation based on the synchrotron and inverse Compton (IC) emission mechanisms. Methods. During the radio-to-optical monitoring provided by the GASP-WEBT, AGILE observed the source with its two co-aligned imagers, the gamma-ray imaging detector (GRID) and the hard X-ray imager (SuperAGILE), which are sensitive in the $30 \mathrm{MeV}-30 \mathrm{GeV}$ and 18-60 keV energy bands, respectively.

Results. In the period 1-16 March 2008, AGILE detected $\gamma$-ray emission from PKS 1510-089 at a significance level of 6.2- $\sigma$ with an average flux over the entire period of $(84 \pm 17) \times 10^{-8}$ photons $\mathrm{cm}^{-2} \mathrm{~s}^{-1}$ for photon energies above $100 \mathrm{MeV}$. After a predefined satellite re-pointing, between 17 and 21 March 2008, AGILE detected the source at a significance level of 7.3- $\sigma$, with an average flux $(E>100 \mathrm{MeV})$ of $(134 \pm 29) \times$ $10^{-8}$ photons $\mathrm{cm}^{-2} \mathrm{~s}^{-1}$ and a peak level of $(281 \pm 68) \times 10^{-8}$ photons $\mathrm{cm}^{-2} \mathrm{~s}^{-1}$ with daily integration. During the observing period January-April 2008, the source also showed an intense and variable optical activity, with several flaring episodes and a significant increase in the flux was observed at millimetric frequencies. Moreover, in the X-ray band, the Swift/XRT observations seem to show a harder-when-brighter behavior of the source spectrum.

Conclusions. The flat spectrum radio quasar PKS 1510-089 showed strong activity between January and April 2008, with episodes of rapid variability from radio to $\gamma$-ray energy bands, in particular with a rapid $\gamma$-ray flaring episode. The spectral energy distribution of mid-March 2008 is modeled with a homogeneous one-zone synchrotron self Compton (SSC) emission plus contributions from inverse Compton scattering of external photons from both the accretion disk and the broad line region. Indeed, some features in the optical-UV spectrum seem to indicate Seyfert-like components such as the little and the big blue bumps.
\end{abstract}

Key words. gamma-rays: observations - radiation mechanisms: non-thermal - quasars: individual: PKS 1510-089

\section{Introduction}

Blazars are a subclass of AGN characterized by the emission of strong nonthermal radiation across the entire electromagnetic spectrum, from radio to very high energies. Their observational properties include irregular, rapid, and often very high

* The radio-to-optical data presented in this paper are stored in the GASP-WEBT archive; for questions regarding their availability, please contact the WEBT President Massimo Villata. variability, apparent super-luminal motion, flat radio spectrum, high and variable polarization at radio and optical frequencies. These features are interpreted as the result of the emission of electromagnetic radiation from a relativistic jet that is viewed closely aligned to the line of sight (Blandford \& Rees 1978; Urry \& Padovani 1995). The spectral energy distribution (SED) of blazars is typically double-humped with a first peak occurring in the IR/optical band in the so-called red blazars (including flat spectrum radio quasars, FSRQs, and low-energy peaked 
BL Lacs, LBLs) and at UV/X-rays in the so-called blue blazars (including high-energy peaked BL Lacs, HBLs). The first peak is commonly interpreted as synchrotron radiation from highenergy electrons in a relativistic jet. The second component of the SED, peaking at MeV-GeV energies in red blazars and at $\mathrm{TeV}$ energies in blue blazars, is commonly interpreted as inverse Compton (IC) scattering of seed photons by relativistic electrons (Ulrich et al. 1997), although a different origin of highenergy emission has been proposed in hadronic models (see e.g., Böttcher 2007, for a recent review).

PKS $1510-089$ is a nearby $(z=0.361)$ radio-loud, highly polarized quasar (HPQ) already detected also in the MeV-GeV energy band by the EGRET instrument on board CGRO (Hartman et al. 1992). The broadband spectrum of the source is representative of the class of FSRQ with the inverse Compton component dominated by the $\gamma$-ray emission, and the synchrotron emission peaked around IR frequencies, even if it is clearly visible in this source a pronunced UV bump possibly caused by the thermal emission from the accretion disk (Malkan \& Moore 1986; Pian \& Treves 1993).

PKS 1510-089 has been extensively observed and studied in the X-ray band by the satellites EXOSAT (Singh et al. 1990; Sambruna et al. 1994), GINGA (Lawson \& Turner 1997), ROSAT (Siebert et al. 1998), ASCA (Singh et al. 1997), and Chandra (Gambill et al. 2003). The observed X-ray spectrum was very flat in the $2-10 \mathrm{keV}$ band with photon index of $\Gamma \simeq 1.3$, but it was steeper $(\Gamma \simeq 1.9)$ in the ROSAT bandpass (0.1-2.4 keV), suggesting a possible spectral break around $1-2 \mathrm{keV}$. The difference in the photon indices could be caused by a soft X-ray excess. Observations by BeppoSAX (Tavecchio et al. 2000) confirm a possible soft X-ray excess below $1 \mathrm{keV}$. Evidence of a similar soft $\mathrm{X}$-ray excess has been detected in other blazars such as 3C 273, 3C 279, AO 0235+164, and $3 \mathrm{C} 454.3$, even if the origin of this excess is still an open issue.

During August 2006, PKS 1510-089 was observed in a relatively bright state by Suzaku over approximately three days and with Swift 10 times during 18 days as a target of opportunity (ToO) with a total duration of $24.3 \mathrm{ks}$. Suzaku measured a very hard X-ray spectrum $(\Gamma<1.5)$, which seems to exclude models in which X-rays are produced by synchrotron radiation of the secondary ultrarelativistic population of electrons and positrons, as predicted by hadronic models. The Swift/XRT observations instead revealed significant spectral evolution in the $0.3-10 \mathrm{keV}$ energy band on timescales of one week, with the spectrum that becomes harder as the source gets brighter (Kataoka et al. 2008).

Gamma-ray emission from PKS 1510-089 was detected several times by EGRET with an integrated flux above $100 \mathrm{MeV}$ between $(13 \pm 5)$ and $(49 \pm 18) \times 10^{-8}$ photons $\mathrm{cm}^{-2} \mathrm{~s}^{-1}$ and an energy spectrum, integrated over all the EGRET observations, modeled with a power law with photon index $\Gamma=2.47 \pm 0.21$ (Hartman et al. 1999).

In August 2007, AGILE detected an intense $\gamma$-ray activity from PKS 1510-089. In particular, during the period 28 August-1 September 2007 the average $\gamma$-ray flux observed was $F_{E>100 \mathrm{MeV}}=(195 \pm 30) \times 10^{-8}$ photons cm $\mathrm{cm}^{-2} \mathrm{~s}^{-1}$ (Pucella et al. 2008). Recently, this source was detected again during high $\gamma$-ray activity states by both the Large Area Telescope (LAT) onboard the Fermi GST (Ciprini et al. 2009; Tramacere 2008; Cutini et al. 2009) and AGILE (D'Ammando et al. 2009; Pucella et al. 2009; Vercellone et al. 2009). The results of the AGILE observations during March 2009 will be published in a forthcoming paper (D'Ammando et al., in preparation).

In this paper we present the analysis of the AGILE data obtained during the observations of PKS 1510-089 from 1 March to 30 March 2008 (see also D’Ammando et al. 2008a). We also present the radio-to-optical monitoring of the GASP-WEBT during the period January-April 2008, and the results of three Swift ToO carried out between 20 and 22 March 2008. This broadband coverage over the entire electromagnetic spectrum allows us to build and study the SED of the source in detail.

The paper is organized as follows. Section 2 describes the AGILE observations, and the corresponding data analysis. Section 3 introduces the Swift data and the relative analysis. Section 4 is dedicated to the results of the GASP-WEBT observations, while in Sect. 5 we discuss the SED, its implication for the emission mechanisms of the source, and finally we draw our conclusions.

Throughout this paper the quoted uncertainties are given at the 1- $\sigma$ level, unless otherwise stated, and the photon indices are parameterized as $N(E) \propto E^{-\Gamma}\left(\mathrm{ph} \mathrm{cm}^{-2} \mathrm{~s}^{-1} \mathrm{keV}^{-1}\right.$ or $\left.\mathrm{MeV}^{-1}\right)$ with $\Gamma=\alpha+1$ ( $\alpha$ is the spectral index). We adopt a luminosity distance of $d_{L}=1915 \mathrm{Mpc}$ for PKS 1510-089, assuming $z=$ 0.361 and a $\Lambda \mathrm{CDM}$ cosmology with $H_{0}=71 \mathrm{Km} \mathrm{s}^{-1} \mathrm{Mpc}^{-1}$, $\Omega_{\mathrm{m}}=0.27$, and $\Omega_{\Lambda}=0.73$.

\section{AGILE data}

\subsection{Observation of PKS 1510-089}

The AGILE satellite (Tavani et al. 2008, 2009) is an Italian Space Agency (ASI) Mission devoted to high-energy astrophysics, with four active detectors capable of observing cosmic sources simultaneously in X-ray and $\gamma$-ray energy bands.

The gamma-ray imaging detector (GRID) consists of a combination of a pair-production silicon tracker (ST; Prest et al. 2003; Barbiellini et al. 2001), sensitive in the energy range $30 \mathrm{MeV}-30 \mathrm{GeV}$, a non-imaging $\mathrm{CsI}(\mathrm{Tl})$ mini-calorimeter (MCAL; Labanti et al. 2009) sensitive in the 0.3-100 MeV energy band, and a segmented anti-coincidence system (ACS) made of plastic scintillator layers that surround all active detectors (Perotti et al. 2006). A co-aligned coded-mask hard X-ray imager (SuperAGILE; Costa et al. 2001; Feroci et al. 2007) ensures coverage in the $18-60 \mathrm{keV}$ energy band.

The AGILE observations of PKS 1510-089 were performed from 1 March 2008 12:45 UT to 21 March 2008 2:04 UT, for a total of $211 \mathrm{~h}$ of effective exposure time. In the first period, between 1 and 16 March, the source was located $\sim 50^{\circ}$ off the AGILE pointing direction. In the second period between 17 and 21 March after a satellite re-pointing, the source was located at $\sim 40^{\circ}$ off-axis. Finally, after a gap of 4 days of observation due to technical maintenance of the satellite, the source was observed at $\sim 50^{\circ}$ off axis between 25 March 13:09 UT and 30 March 10:29 UT. Unfortunately, during the observation the source was substantially off-axis in the field of view of SuperAGILE.

\subsection{Data reduction and analysis}

AGILE-GRID data were analyzed, starting from the Level-1 data, using the AGILE Standard Analysis Pipeline (see Vercellone et al. 2008, for a detailed description of the AGILE data reduction). An ad-hoc implementation of the Kalman Filter technique is used for track identification and event-direction reconstruction in detector coordinates and subsequently a quality flag is assigned to each GRID event, depending on whether it is recognized as a confirmed gamma-ray event, a charged particle event, a single-track event, or as having an uncertain nature.

After the creation of the event files, the AGILE Scientific Analysis Package can be run. Counts, exposure, and Galactic 
background $\gamma$-ray maps were generated with a bin size of $0.25^{\circ} \times$ $0.25^{\circ}$ for photons with energy $E>100 \mathrm{MeV}$.

To reduce the particle background contamination, we selected only the events flagged as confirmed $\gamma$-ray events, so all events collected during the South Atlantic Anomaly were rejected. We also rejected all the $\gamma$-ray events whose reconstructed directions form angles with the satellite-Earth vector smaller than $80^{\circ}$, in order to reduce the $\gamma$-ray Earth albedo contamination. The most recent version (BUILD-16) of the Calibration files at the time of writing (publicly available at the ASI Science Data Center (ASDC) site ${ }^{1}$ ) and of the $\gamma$-ray diffuse emission model (Giuliani et al. 2004) is used. We ran the AGILE maximum likelihood procedure with a radius of analysis of $10^{\circ}$, on the whole observing period, in order to obtain the average flux in the $\gamma$-ray band and estimate the diffuse parameters used to measure the daily fluxes, according to the procedure described in Mattox et al. (1993).

\subsection{Results}

During the period 1-16 March 2008, AGILE-GRID detected $\gamma$-ray emission from a position consistent with the powerful $\gamma$-ray quasar PKS 1510-089 at a significance level of 6.2- $\sigma$ with an average flux over the entire period of $(84 \pm 17) \times 10^{-8}$ photons $\mathrm{cm}^{-2} \mathrm{~s}^{-1}$ for photon energies above $100 \mathrm{MeV}$.

Instead, in the period 17-21 March 2008, AGILE detected $\gamma$-ray emission from a position consistent with the source at a significance level of 7.3- $\sigma$. The AGILE 95\% maximum likelihood contour level baricenter of the source is $l=351.49^{\circ}, b=$ $40.07^{\circ}$, with a distance between this position and the radio position $\left(l=351.29^{\circ}, b=40.14^{\circ}\right)$ of $0.17^{\circ}$. The overall AGILE error circle, taking both statistical and systematic effects into account, has a radius $r=0.50^{\circ}$. The average flux above $100 \mathrm{MeV}$ during this second period, with the source located $\sim 40^{\circ}$ off the AGILE pointing direction, was $(134 \pm 29) \times$ $10^{-8}$ photons $\mathrm{cm}^{-2} \mathrm{~s}^{-1}$. The peak level of activity with daily integration was $(281 \pm 68) \times 10^{-8}$ photons $\mathrm{cm}^{-2} \mathrm{~s}^{-1}$, showing an increase of a factor two in one day and at least three in two days, as the source had not been detected for some days after two episodes of medium intensity. After the sudden increase, the flux rapidly decreased around 19 March 2008.

When fitting the data relative to the period 17-21 March with a simple power law model, we obtain a photon index of $\Gamma=$ $1.81 \pm 0.34$. This photon index is calculated with the weighted least squares method, considering for the fit three energy bins: 100-200 MeV, 200-400 MeV, and 400-1000 MeV. The photon index obtained for this second period is consistent within the errors with the one observed by AGILE in August 2007 ( $\Gamma=$ $1.98 \pm 0.27$ ).

Figure 1 shows the $\gamma$-ray light curve between 1 and 21 March 2008 with 2-day resolution for the first period and 1-day for the second period, for photons of energy above $100 \mathrm{MeV}$. The downward arrows represent 2- $\sigma$ upper limits. Upper limits are calculated when the analysis provides a significance of detection $<3-\sigma$ (see Mattox et al. 1996). Finally, in the third period between 25 and 30 March 2008 the source was not detected by the GRID and an upper limit with $95 \%$ confidence level of $54 \times$ $10^{-8}$ photons $\mathrm{cm}^{-2} \mathrm{~s}^{-1}$ is provided.

During August-October 2008, Fermi-LAT detected the source with an average flux for $E>100 \mathrm{MeV}$ of $(55.8 \pm 3.3) \times$ $10^{-8}$ photons $\mathrm{cm}^{-2} \mathrm{~s}^{-1}$ and a peak of intensity of $(165.9 \pm 11.7) \times$ $10^{-8}$ photons $\mathrm{cm}^{-2} \mathrm{~s}^{-1}$ (Abdo et al. 2009). The peak of $\gamma$-ray

${ }^{1}$ http://agile.asdc.asi.it

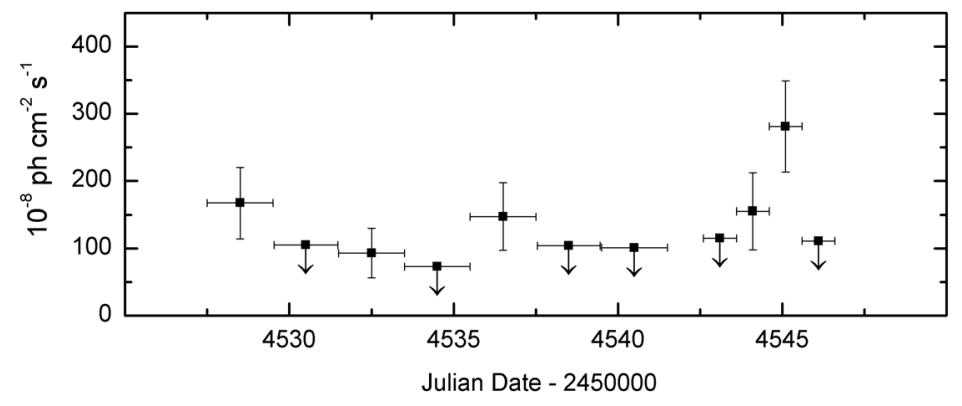

Fig. 1. AGILE-GRID $\gamma$-ray light curve between 1 and 21 March 2008 at 1-day or 2-day resolution for $E>100 \mathrm{MeV}$ with fluxes in units of $10^{-8}$ photons $\mathrm{cm}^{-2} \mathrm{~s}^{-1}$. The downward arrows represent $2-\sigma$ upper limits.

emission corresponds to the first flare observed by Fermi-LAT at the end of September 2008 (Tramacere 2008), and the average flux value confirms the flaring state observed by AGILE in mid-March.

Moreover, Fermi-LAT observed a softer photon index for this source in August-October 2008, $\Gamma=2.48 \pm 0.05$ (Abdo et al. 2009), but this value corresponds to an average value over three months of observation in which the source flux was variable, whereas the value reported by us refers to a rapid flaring episode. The value obtained by Fermi-LAT is very similar to what was measured by EGRET averaging over all the observations $(\Gamma=2.47 \pm 0.21)$, confirming that the average spectral indexes are softer than those measured during short flaring states. The difference between the value obtained by AGILE and Fermi could also be partially due to the different bandpasses of the two intruments.

\section{SWIFT observations}

The NASA Swift gamma-ray burst mission (Gehrels et al. 2004), performed three ToO observations of PKS 1510-089 in three consecutive days with the first occurring on 20 March 2008. The three observations were performed using all three onboard experiments: the X-ray Telescope (XRT; Burrows et al. 2005, 0.2-10 keV), the UV and Optical Telescope (UVOT; Roming et al. 2005, 170-600 nm) and the coded-mask Burst Alert Telescope (BAT; Barthelmy et al. 2005, 15-150 keV). The hard X-ray flux of this source is below the sensitivity of the BAT instrument for so short exposure, so the data from this instruments will not be used.

\subsection{Swift/XRT data}

The XRT data were processed with standard procedures (xrtpipeline v0.12.0), and the filtering and screening criteria were applied by means of the FTOOLS in the Heasoft package v6.5. Given the low rate of PKS 1510-089 during the three observations $\left(<0.5\right.$ count $^{-1}$ in the $0.2-10 \mathrm{keV}$ range), we only considered photon counting (PC) data for our analysis, and further selected XRT grades 0-12 (according to Swift nomenclature, see Burrows et al. 2005). No pile-up correction was necessary. The ancillary response files were generated with the task xrtmkarf, applying corrections for the PSF losses and CCD defects, and we used the latest spectral redistribution matrices (RMF, v011) in the calibration database maintained by HEASARC. The adopted energy range for spectral fitting is $0.3-10 \mathrm{keV}$, and all data were rebinned with a minimum of 
Table 1. Observation log and fitting results of Swift/XRT observations of PKS 1510-089, with power-law model with $N_{\mathrm{H}}$ fixed to Galactic absorption.

\begin{tabular}{cccccc}
\hline \hline $\begin{array}{c}\text { Observation } \\
\text { date }\end{array}$ & $\begin{array}{c}\text { Exposure time } \\
(\mathrm{s})\end{array}$ & $\begin{array}{c}\text { Counts } \\
(0.2-10 \mathrm{keV})\end{array}$ & $\begin{array}{c}\text { Flux 0.3-10 keV } \\
\mathrm{erg} \mathrm{cm}^{-2} \mathrm{~s}^{-1}\end{array}$ & $\begin{array}{c}\text { Photon index } \\
\Gamma\end{array}$ & $\chi_{\text {red }}^{2}$ (d.o.f.)/C-stat $(\%)^{b}$ \\
\hline 20-Mar-2008 & 1961 & 306 & $12.20_{-1.65}^{+1.65} \times 10^{-12}$ & $1.16 \pm 0.16$ & $0.87(13)$ \\
21-Mar-2008 & 1966 & 189 & $8.77_{-1.39}^{+1.33} \times 10^{-12}$ & $1.53 \pm 0.17$ & $467(48.2)^{b}$ \\
22-Mar-2008 & 1885 & 261 & $9.48_{-1.14}^{+1.13} \times 10^{-12}$ & $1.41 \pm 0.19$ & $1.34(11)$ \\
\hline
\end{tabular}

${ }^{a}$ Unabsorbed flux; ${ }^{b}$ cash statistic (C-stat) and percentage of Montecarlo realizations that had statistic $<$ C-stat, performing $10^{4}$ simulations.

20 counts per energy bin to use the $\chi^{2}$ minimization fitting technique. An exception is the observation of 21 March 2008, when the number of counts was so low that the Cash statistic (Cash 1979) on ungrouped data was used. Swift/XRT uncertainties are given at $90 \%$ confidence level for one interesting parameter, unless otherwise stated.

Spectral analysis was performed using the XSPEC fitting package 12.4.0 (Arnaud et al. 1996). We fitted the spectra with a power-law model with Galactic absorption fixed to $N_{\mathrm{H}}=$ $6.89 \times 10^{20} \mathrm{~cm}^{-2}$ (Kalberla et al. 2005). Table 1 summarizes the most important information on XRT observations and the relative spectral fit parameters.

A variability of about $30 \%$ in the X-ray flux of the source was observed on a timescale of one day. Notwithstanding the uncertainties due to the errors on fluxes and photon indexes, the XRT data seem to indicate that the X-ray spectrum becomes harder when the source gets brighter, confirming the behavior already observed in this source by Kataoka et al. (2008) during the Swift/XRT observations carried out in August 2006. This is a trend often observed in HBL (see e.g. Massaro et al. 2008; Tramacere et al. 2007; Kataoka et al. 1999), but quite rare for quasar-hosted blazars such as PKS 1510-089. For instance, $3 \mathrm{C} 454.3$ shows approximately the same spectral slope in different brightness states (see e.g. Raiteri et al. 2007; Raiteri et al. 2008).

\subsection{Swift/UVOT data}

During the three Swift pointings, the UVOT instrument (Poole et al. 2008) observed PKS $1510-089$ in all its optical ( $V, B$, and $U$ ) and UV (UVW1, UVM2, and UVW2) photometric bands. Data were reduced with the uvotmaghist task of the HEASOFT package. Source counts were extracted from a circular region of 5 arcsec radius, centered on the source, while the background was estimated from a surrounding annulus with 8 and 18 arcsec radii. In the first two days, only one exposure per filter was available, while three exposures per filter were acquired on the last day. With the only exception of UVM2, the source brightness turned out to be quite stable in all the UVOT bands, with variations of a few hundredth of mag, well inside the typical UVOT data uncertainty of $0.1 \mathrm{mag}$ due to both systematic and statistical errors. Average values are: $V=16.94, B=17.19$, $U=16.31, \mathrm{UV} W 1=16.64$, and UVW2 $=16.55$. The UVM2 frames have low signal-to-noise ratios, thus the source magnitude in this band presents a larger dispersion. The average value is $\mathrm{UVM} 2=16.47 \pm 0.14$.

\section{Radio-to-optical observations by the GASP}

PKS $1510-089$ is one of the $28 \gamma$-ray-loud blazars that are regularly monitored by the GLAST-AGILE Support Program
(GASP; Villata et al. 2008) of the Whole Earth Blazar Telescope (WEBT). Optical and near-IR data are collected as already calibrated magnitudes, according to a common choice of photometric standards from Raiteri et al. (1998). Radio data are provided as calibrated flux densities. The reference optical band for the GASP is the $R$ band. The corresponding light curve in JanuaryApril 2008 is shown in the top panel of Fig. 2, with the data provided by the following observatories: Abastumani, Calar Alto ${ }^{2}$, Crimean, Lowell (Perkins), Lulin, Roque de los Muchachos (KVA and Liverpool), San Pedro Martir, St. Petersburg, Torino. The source showed intense activity during the entire considered period, with several episodes of fast variability. At the beginning of the optical observing season, the January observations indicate that the source was in a faint state, around $R=16.6$. A fast brightening of $\sim 1.3 \mathrm{mag}$ in 8 days led the source to $R=15.3$ on 15 February. This was followed by a $\sim 0.6$ mag dimming in 4 days. Other minimum brightness states were observed on 23 March and in late April, while peaks were detected on March 29 and April 11.

Near-IR data in the $J H K$ bands were taken at Campo Imperatore and Roque de los Muchachos (Liverpool). Millimetric flux densities at 345 and $230 \mathrm{GHz}$ came from the SubMillimeter Array (SMA) on Mauna Kea. Centimetric radio data were acquired at Medicina (22 and $8 \mathrm{GHz}$ ), Noto (43 GHz), and UMRAO (14.5, 8.0, and 4.8 GHz). In Fig. 1 the source radio behavior in different bands is compared to the optical one (top panel). The light curve at high radio frequencies (230-345 GHz) suggests that the mechanism producing the flaring events observed in the optical band in the second half of February and in late March-April 2008 also interested the millimetric emitting zone, with some delay. An estimate of this delay is hampered by the limited data sampling. At lower radio frequencies (22-43 GHz), a hint of flux increase is visible in the second part of the light curve, while the radio flux at $5-15 \mathrm{GHz}$ shows no trend at all. This suggests that the jet regions that are responsible for the emission at the longest radio wavelengths are not affected by the flaring mechanism.

\section{Discussion and conclusions}

In the past two years, PKS 1510-089 has shown very high activity in $\gamma$-ray band with several flaring episodes (see Pucella et al. 2008; Tramacere 2008; Ciprini et al. 2009; D'Ammando et al. 2009; Pucella et al. 2009; Vercellone et al. 2009; Cutini et al. 2009). During the period January-April 2008, the source showed a high variability over the whole electromagnetic spectrum from radio to $\gamma$-rays, with several flaring episodes in the optical band and a rapid and intense flare detected in the $\gamma$-ray band in mid-March.

2 Calar Alto data were acquired as part of the MAPCAT (Monitoring AGN with Polarimetry at the Calar Alto Telescopes) project. 


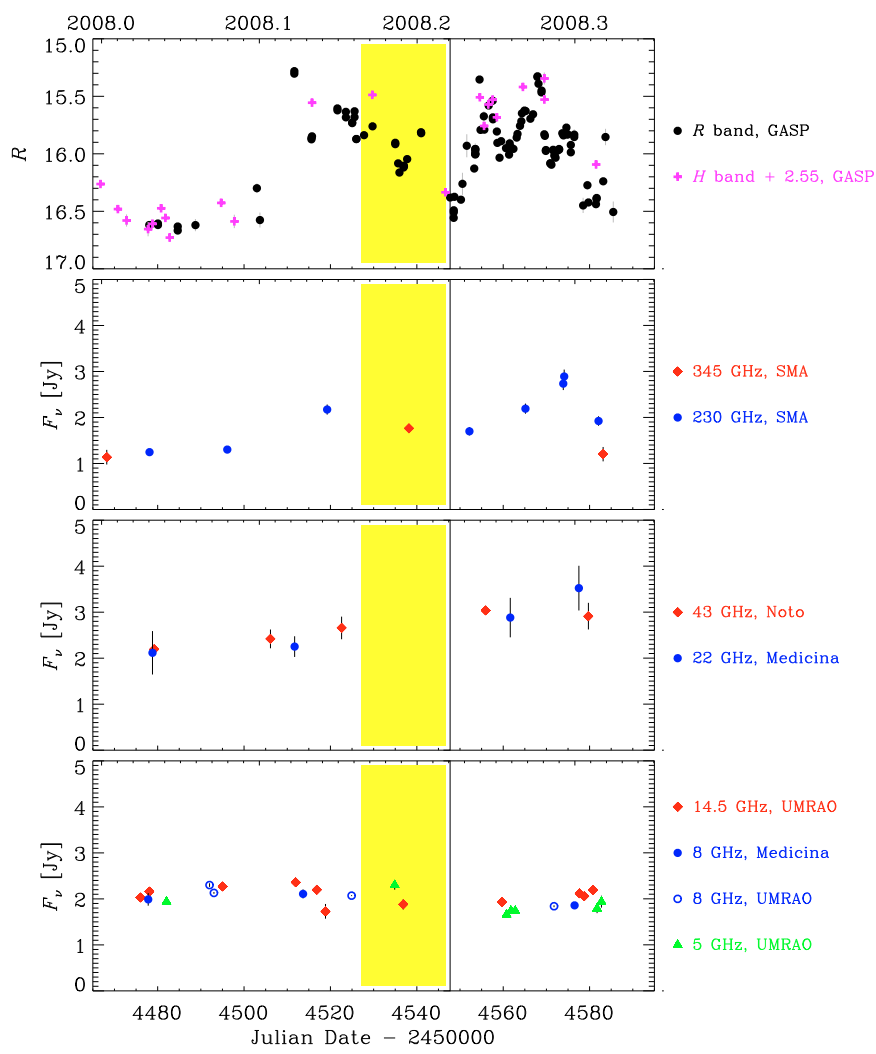

Fig. 2. Optical light curve of PKS 1510-089 (top panel) obtained by the GASP-WEBT during the period January-April 2008 compared to its radio flux densities at different frequencies. The vertical bar indicates the time of the Swift observations. The yellow shaded region marks the period also covered by the AGILE observation.

\subsection{Modeling the spectral energy distribution}

Figure 3 shows the SED for the AGILE-GRID observation period 17-21 March 2008, including quasi-simultaneous optical and radio data by GASP and UVOT and X-ray data by Swift. Since the source brightness over the three days of UVOT observations remained stable, we built a unique SED for the whole period, including contemporaneous data at other frequencies, in particular radio-to-optical data from the GASP. The optical and near-IR data were acquired exactly in the period of the UVOT observations: one $R$-band datum from Roque (KVA) and $J, H$, and $K$ data from Campo Imperatore. The UVOT and GASP magnitudes were corrected for Galactic extinction by adopting $A_{\mathrm{B}}=0.416 \mathrm{mag}$, and deriving the values in the other bands according to Cardelli et al. (1989). To convert magnitudes into fluxes, we assumed the zero-mag fluxes by Poole et al. (2008) and Bessel et al. (1998).

Radio light curves are not sampled any more than was the $R$-band one (see Fig. 2), therefore in the SED we show the highfrequency radio data $(43-345 \mathrm{GHz})$ taken within a week from the UVOT observations, while the low-frequency data points were obtained by interpolating between the closest data preceeding and following the UVOT observations. This is justified by the smooth behavior of the low-frequency radio light curves.

The dip in the SED corresponding to the UVW1 frequency must be regarded with caution, since it is also found for other blazars with different redshifts and could be systematic. Observations performed by Swift and the GASP in March and June 2007, when the source was nearly at the same brightness level, showed the same shape in the near-IR-to-UV part of
Table 2. Parameters for the model used to explain the SED of PKS 1510-089 during the $\gamma$-ray flare of 18, 19 March 2008.

\begin{tabular}{ccr}
\hline \hline Parameter & Value & Units \\
\hline$p_{1}$ & 2.2 & \\
$p_{2}$ & 4.6 & \\
$\gamma_{\min }$ & 30 & \\
$\gamma_{\mathrm{b}}$ & 290 & \\
$\gamma_{\max }$ & 5200 & \\
$K$ & 75 & $\mathrm{~cm}^{-3}$ \\
$R$ & 10 & $10^{15} \mathrm{~cm}$ \\
$B$ & 3.5 & $\mathrm{G}$ \\
$\delta$ & 20.26 & \\
$L_{\mathrm{d}}$ & 5 & $10^{45} \mathrm{erg} \mathrm{s}^{-1}$ \\
$\theta$ & 2.86 & degrees \\
$\Gamma$ & 18 & \\
\hline
\end{tabular}

the SED. A similar trend characterized the optical-UV SED of August 2006 shown by Kataoka et al. (2008). We notice that the shape of the SED in the optical band may be affected by the flux contribution of broad emission lines, including the little blue bump (Neugebauer et al. 1979; Smith et al. 1988). For the SED we used the Swift/XRT data collected on 20 March, the observation closest to the $\gamma$-ray flare and during which the higher X-ray flux was observed.

To model the SED we used a homogeneous one-zone synchrotron self Compton (SSC; Marscher \& Gear 1985; Maraschi et al. 1992; Bloom \& Marscher 1996) model, plus the contribution of external Compton scattering of both direct-disk radiation (ECD; Dermer et al. 1992) and photons from the broad line region (BLR) clouds (ECC; Sikora et al. 1994). The strong thermal features usually observed in FSRQs (and in this blazar in particular, see Neugebauer et al. 1978; Smith et al. 1988) at optical/UV frequencies suggest that the environment is rich in the soft photons produced by the accretion disk and/or reprocessed by the BLR. This implies that the energy density of the external soft radiation is much higher than for the synchrotron radiation; therefore, during the $\gamma$-ray flares in FSRQs, the most important processes are the ECC and ECD, and the $\gamma$-ray photon index could be determined by the dominant contribution of the two.

We consider a moving spherical blob of radius $R$, filled by relativistic electrons and embedded in a random magnetic field. We assume that the electron energy density distribution is described by a broken power law:

$n_{\mathrm{e}}(\gamma)=\frac{K \gamma_{\mathrm{b}}^{-1}}{\left(\gamma / \gamma_{\mathrm{b}}\right)^{p_{1}}+\left(\gamma / \gamma_{\mathrm{b}}\right)^{p_{2}}}$

where $\gamma$ is the electron Lorentz factor assumed to vary between $\gamma_{\min }$ and $\gamma_{\max }, p_{1}$ and $p_{2}$ are pre- and post-break electron distribution spectral indexes, respectively, and $\gamma_{\mathrm{b}}$ is the break energy Lorentz factor. We assume that the blob contains a comoving random average magnetic field $B$ with a bulk Lorentz factor $\Gamma$ at an angle $\theta$ with respect to the line of sight. The relativistic Doppler beaming factor is then $\delta=[\Gamma(1-\beta \cos \theta)]^{-1}$, and $K$ is the normalization density parameter into the blob.

We have chosen an angle of view of $0.05 \mathrm{rad}$ in agreement with both the apparent jet velocities derived from multiepoch Very Long Baseline Array (VLBA) observations of the source (Homan et al. 2001; Wardle et al. 2005; Jorstad et al. 2005; Lister et al. 2009) and the value used by Kataoka et al. (2008).

The short time variability observed in $\gamma$-ray band constrains the size of the emitting region to $R<c \Delta t_{\mathrm{var}} \delta /(1+z)=3.86 \times$ $10^{16} \mathrm{~cm}$, where $\Delta t_{\mathrm{var}}$ is the observed variation time. An accretion 


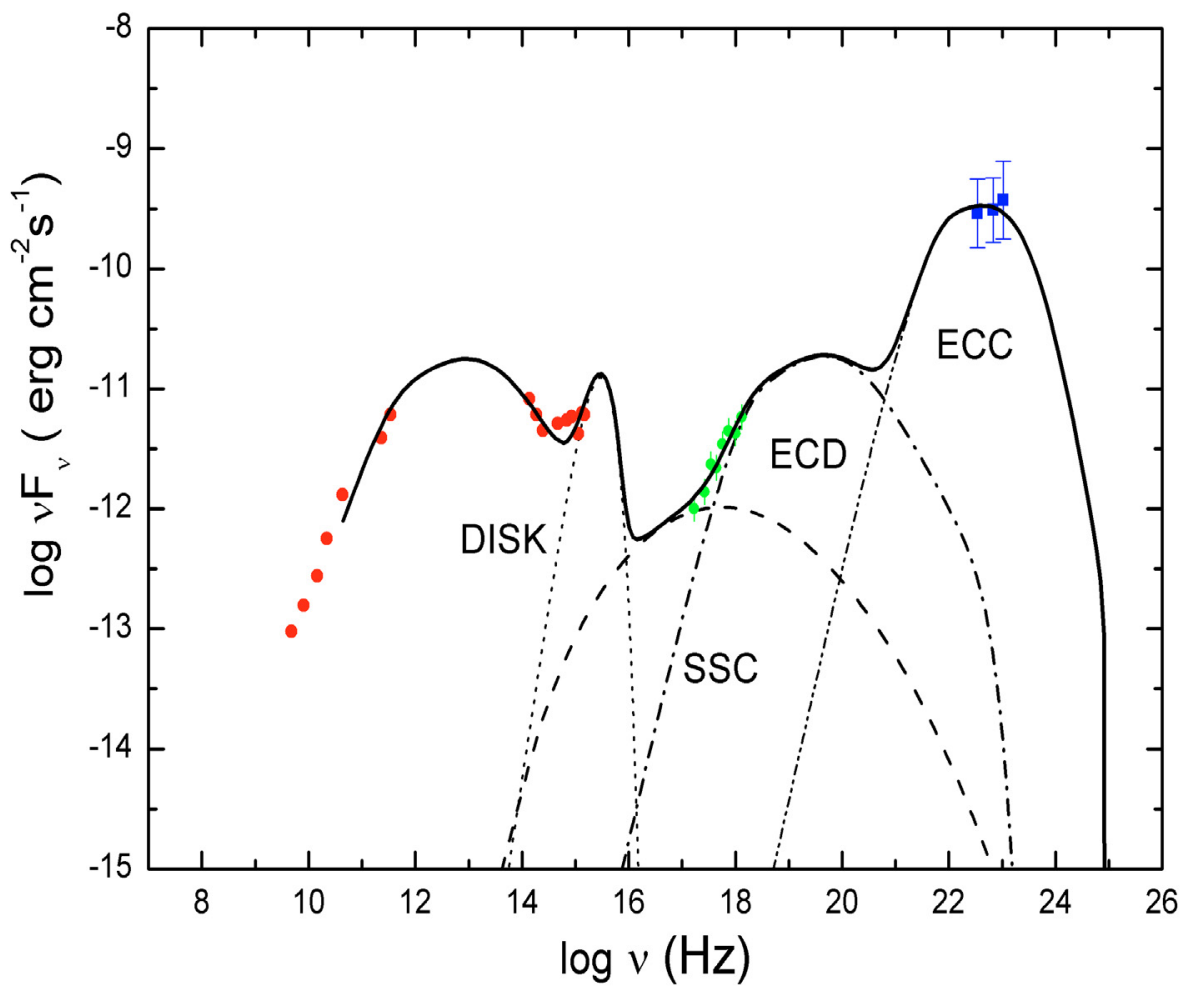

Fig. 3. Spectral energy distribution of PKS 1510-089 for the AGILE-GRID observation of 17-21 March 2008, including quasisimultaneous GASP radio-to-optical data, the Swift/UVOT data of 20-22 March and the Swift/XRT data of 20 March. The dotted, dashed, dot-dashed, and double-dot-dashed lines represent the accretion disk black body, the SSC, the ECD, and the ECC radiation, respectively. disk characterized by a black body spectrum with a luminosity of $5 \times 10^{45} \mathrm{erg} \mathrm{s}^{-1}$, as estimated with UV observations by Pian \& Treves (1993), at $0.05 \mathrm{pc}$ from the blob is assumed as one of the sources of external target photons. We also assumed a BLR at $0.2 \mathrm{pc}$, reprocessing $10 \%$ of the irradiating continuum. The IC spectra derived from the approximation of the BLR radiation as a black body reproduces more refined spectra calculated by Tavecchio et al. (2008) quite well when taking the more accurate shape of the BLR into account.

Assuming a model with synchrotron, SSC and EC components plus the contribution from the accretion disk radiation, the SED of mid March 2008 can be represented well with input parameters summarized in Table 2. In the choice of the parameters we were guided by the knowledge of the angle of view, the disk luminosity and the simultaneous observations of the synchrotron and IC peak regions (therefore of the synchrotron and IC peak frequencies and luminosities). In addition, the minimum variability timescale gives an indication of the size of the emitting region. However, even if these quantities are quite tightly constrained by fitting the whole SED, the choice of some parameters is not unique because the simultaneous presence of the synchrotron, SSC, ECC, and ECD components leads to a possible partial degeneration of the parameters.

\subsection{X-ray spectral evolution}

The spectral evolution detected in X-rays by Swift in just two days, soon after the $\gamma$-ray flaring episode is another hint of the rapid change in activity by this source. Usually in FSRQs as PKS 1510-089 only little variability is observed in X-rays on short timescales from hours to days, and also on longer timescales the X-ray spectral shape is almost constant with only small variations. The photon indices measured by Swift/XRT in March 2008, in particular during the first observation, tend to be lower than usually observed in FSRQs $\left(\left\langle\Gamma_{[0.1-2.4]}\right\rangle=1.76 \pm 0.06\right.$ and $\left\langle\Gamma_{[2-10]}\right\rangle=1.65 \pm 0.04$, Donato et al. 2001) and are more like those observed in some high-redshift quasars (such as RBS 315 , Tavecchio et al. 2007, and Swift J0746+2548, Sambruna et al. 2007). The hard photon indices of high-redshift blazars could be interepreted in terms of absorption by warm plasma in the region surrounding the source, in agreement with a scenario where in the early evolution phases the quasars are substantially obscured by gas subsequently expelled from the host galaxy by powerful winds (see e.g. Fabian 1999). However, considering the low redshift, this interpretation is unlikely for this source.

Instead, the X-ray spectral evolution observed by Swift/XRT could be caused by the contamination of an additional component below $\sim 2 \mathrm{keV}$ : the soft $\mathrm{X}$-ray excess. In fact, previous observations with Chandra (Gambill et al. 2004) and Suzaku (Kataoka et al. 2008) seem to indicate the soft X-ray excess in the spectrum of PKS 1510-089. The soft X-ray excess is an emission in excess of the extrapolation of the power-law component dominating at higher energies, but the origin of this excess in AGNs is still an open issue (see e.g. D'Ammando et al. 2008, for a detailed discussion). In the past, it was often associated with the thermal emission of the accretion disk and then related to the big blue bump. However, it has recently been shown that modeling the soft X-ray excess in non-blazar AGNs with a thermal component yields a remarkably constant disk temperature, around $0.1-0.2 \mathrm{keV}$, regardless of the central black hole mass and luminosity (Gierlinsky \& Done 2004; Crummy et al. 2006). Also in Kataoka et al. (2008) the soft X-ray excess is tentatively described by a black body with temperature $k T \simeq 0.2 \mathrm{keV}$. This result is difficult to explain in any model for the soft excess related to disk continuum emission, as the temperature in any disk model is expected to vary with both the black hole mass and the accretion rates.

For FSRQs one possible theoretical explanation is that the soft X-ray excess is a bulk Comptonization feature produced by cold plasma accelerated in a jet (Celotti et al. 2007), even if this 
feature has never been positively observed until now. In BL Lac objects the radiative environment is instead too weak to produce the soft X-ray excess via bulk Compton and the soft X-ray excess is likely related to the high-energy tail of the synchrotron emission.

The change in the photon index observed during the Swift/XRT observations could stem from the spectral shape of the inverse Compton component in X-ray remaining roughly constant, but the amount of contamination from the soft excess emission varies. The contribution of the soft X-ray excess would be more significant when the source gets fainter, affecting the spectrum at higher energies. Unfortunately, the brief exposure of the Swift observation does not allow a detailed spectral modeling of this feature.

One possibility for the origin of this hard power law in PKS 1510-089 is that the photon index observed in X-rays results from the combination of the synchrotron self Compton and external Compton emission, hence from the mismatch of the spectral slopes of these two components, not to a real soft X-ray excess. This is the solution that the data presented in this paper would favor. In this context, the spectral evolution during the three Swift/XRT observations could come from the change in contribution of one of the two components and therefore from a different variability of the SSC and EC components.

\subsection{Thermal emission components}

Even if the SED of the blazars are usually dominated by the beamed nonthermal jet radiation, some of them show the signature of Seyfert-like features such as the little and the big blue bumps. The little blue bump is usually observed in quasars between $\sim 2000$ and $\sim 4000 \AA$ in the rest frame and likely stems from the contribution of FeII and MgII emission lines and the Balmer continuum produced in the broad line region (Wills et al. 1985). The big blue bump instead is associated with a rise in the $U V$ band commonly interpreted as thermal emission from the accretion disk (see e.g. Laor 1990). Evidence of these thermal components has been found in other quasar-like blazars, such as 3C 273 (Grandi and Palumbo 2004; Türler et al. 2006), 3C 279 (Pian et al. 1999) and 3C 454.3 (Raiteri et al. 2007).

The presence of the emission by the accretion disk is consistent with the scenario in which the seed photons for the IC producing the $\gamma$-rays are external to the relativistic jet, but it is usually not observed because hidden by the beamed variable synchrotron emission. That the synchrotron component of PKS 1510-089 peaks around $10^{13} \mathrm{~Hz}$ (see Bach et al. 2007; Nieppola et al. 2008) allows us to observe these thermal features in this source. In fact, around $10^{15} \mathrm{~Hz}$, a rising emission is visible in the spectrum and is likely a manifestation of the big blue bump produced by the accretion disk, as already discussed for this source by Malkan \& Moore (1986) and Pian \& Treves (1993); moreover, a hint of the little blue bump seems to appear in the SED of the source at $10^{14.5} \mathrm{~Hz}$.

Given the redshift of PKS 1510-089, the $\mathrm{H} \alpha, \mathrm{H} \beta$, FeII, and MgII lines mostly contribute to the observed spectrum between $10^{14.2}$ and $10^{14.8} \mathrm{~Hz}$, and together with the disk emission, could explain the excess of emission observed around $10^{14.5} \mathrm{~Hz}$ and not modeled from the other components represented in the SED. Moreover these non-jet components in the blue part of the spectrum of this blazar have already been observed by Neugebauer et al. (1979) and Smith et al. (1988) and it agrees with the redderwhen-brighter behavior shown by the $B-R$ index versus $R$-band

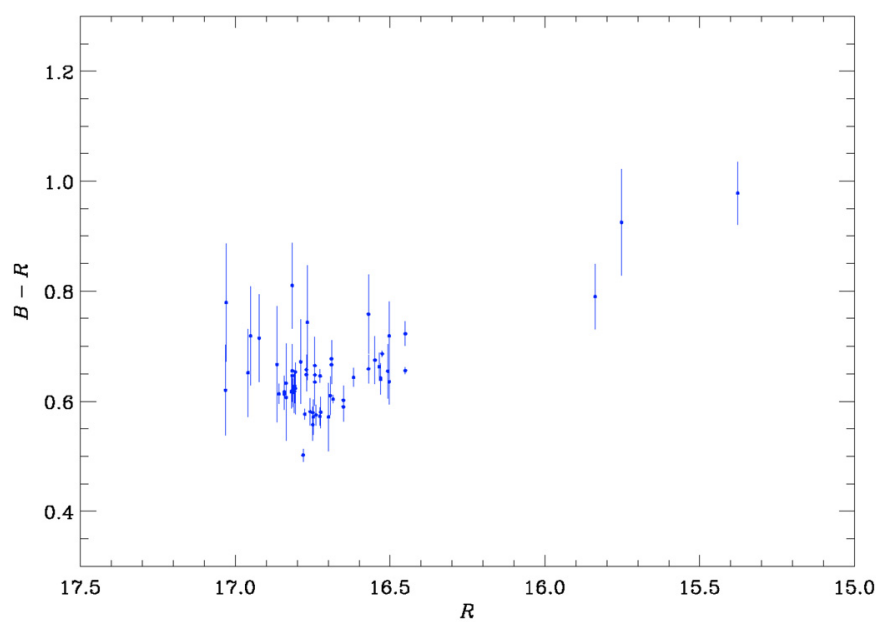

Fig. 4. $B-R$ color index versus $R$-band magnitude for PKS 1510-089 obtained with archive data of the Torino Observatory.

plot in Fig. 4. The plot has been obtained with archive data stored at the Torino Observatory.

\subsection{Energetics and alternative model}

Finally, to estimate the energetics of PKS 1510-089, we computed the isotropic luminosity in the $\gamma$-ray band, comparing it with the Eddington and bolometric luminosity and the total power transported by the jet. For a given source with redshift $z$, the isotropic emitted luminosity in the energy band $\epsilon$ is defined as

$L(z)_{\epsilon}=\frac{4 \pi F d_{1}^{2}(z)}{(1+z)^{\left(1-\alpha_{\gamma}\right)}}$

where, in our case, $\epsilon$ is the $\gamma$-ray energy band with $E_{\min }=$ $100 \mathrm{MeV}$ and $E_{\max }=10 \mathrm{GeV}, \alpha_{\gamma}=\Gamma-1, F$ the $\gamma$-ray energy flux between $E_{\min }$ and $E_{\max }$, calculated from the photon flux $F_{\gamma}$ $(E>100 \mathrm{MeV})$ as suggested by Ghisellini et al. (2009):

$F=1.6 \times 10^{-12} \frac{\alpha_{\gamma} F_{\gamma}}{1-\alpha_{\gamma}}\left[100^{1-\alpha_{\gamma}}-1\right]$.

The luminosity distance is given by

$\mathrm{d}_{1}\left(z_{1}, z_{2}\right)=\left(1+z_{2}\right)^{2} \times \frac{c / H_{0}}{1+z_{2}} \int_{z_{1}}^{z_{2}}[E(z)]^{-1} \mathrm{~d} z$

where $\mathrm{z}_{1}=0, \mathrm{z}_{2}=\mathrm{z}_{\mathrm{src}}$, and

$E(z)=\sqrt{\Omega_{\mathrm{m}}(1+z)^{3}+\left(1-\Omega_{\mathrm{m}}-\Omega_{\Lambda}\right)(1+z)^{2}+\Omega_{\Lambda}}$,

where $H_{0}$ is the Hubble constant, and $\Omega_{m}$ and $\Omega_{\Lambda}$ are the contribution of the matter and of the cosmological constant, respectively, to the density parameter. Using a luminosity distance $d_{L}=1915 \mathrm{Mpc}$ and the average $\gamma$-ray flux observed by the AGILE-GRID during 17-21 March 2008, we obtained an isotropic luminosity $L_{\gamma}^{\text {iso }}=5.3 \times 10^{47} \mathrm{erg} \mathrm{s}^{-1}$ for PKS $1510-089$ $(z=0.361)$.

The power carried by the jet in the form of magnetic field $\left(L_{\mathrm{B}}\right)$, cold protons $\left(L_{\mathrm{p}}\right)$, relativistic electrons $\left(L_{\mathrm{e}}\right)$, and produced radiation $\left(L_{\mathrm{rad}}\right)$, are

$L_{\mathrm{p}}=\pi R^{2} \Gamma^{2} c \int\left[N(\gamma) m_{\mathrm{p}} c^{2} \mathrm{~d} \gamma\right]=3.6 \times 10^{45} \mathrm{erg} \mathrm{s}^{-1}$ 
$L_{\mathrm{e}}=\pi R^{2} \Gamma^{2} c \int\left[N(\gamma) \gamma m_{\mathrm{e}} c^{2} \mathrm{~d} \gamma\right]=1.5 \times 10^{44} \mathrm{erg} \mathrm{s}^{-1}$

$L_{\mathrm{B}}=\pi R^{2} \Gamma^{2} c U_{\mathrm{B}}=1.5 \times 10^{45} \mathrm{erg} \mathrm{s}^{-1}$

$L_{\mathrm{rad}} \simeq L_{\mathrm{iss}} \Gamma^{2} / \delta^{4}=5.3 \times 10^{45} \mathrm{erg} \mathrm{s}^{-1}$,

where $U_{\mathrm{B}}$ is the magnetic energy density. Therefore the total power transported by the jet is $P=L_{\mathrm{B}}+L_{\mathrm{p}}+L_{\mathrm{e}}+L_{\mathrm{rad}}=1.1 \times$ $10^{46} \mathrm{erg} \mathrm{s}^{-1}$.

Assuming a black hole mass $M_{\mathrm{BH}}=4.5 \times 10^{8} M_{\odot}$ for the source (Woo \& Urry 2002), we obtain an Eddington luminosity

$L_{\mathrm{Edd}}=\frac{4 \pi G c m_{H}}{\sigma_{T}} M_{\mathrm{BH}}=5.7 \times 10^{46} \mathrm{erg} \mathrm{s}^{-1}$

to be compared with the bolometric luminosity $L_{\mathrm{bol}}=2.4 \times$ $10^{46} \mathrm{erg} \mathrm{s}^{-1}$ reported in Woo \& Urry (2002).

An alternative theoretical model has recently been proposed by Kataoka et al. (2008) to interpret the data of PKS 1510-089 collected during August 2006, with the high-energy emission originated by the Comptonization of infrared radiation produced by the molecular torus surrounding the central engine and suggesting that the soft X-ray excess could be produced by the IC scattering of external photons by a population of cold electrons, as discussed by Begelman et al. (1987) and Celotti et al. (2007). An accurate theoretical interpretation of the SED is beyond the scope of this paper; however, our data do not seem to rule out this alternative model. In fact, we do not have simultaneous observations in FIR band that can confirm the excess detected by IRAS (Tanner et al. 1996), interpreted by Kataoka et al. as due to dust radiation from the nuclear torus and assumed to be the main source of seed photons for the IC mechanism. Moreover, the bulk Comptonization feature should not be observable during a high activity state of the source, such as observed in midMarch 2008, because overwhelmed by the SSC and ECD emission, therefore this is not the best situation for testing this hypothesis.

Further X-ray observations with XMM-Newton and Suzaku, simultaneously with the optical monitoring by means of the REM Telescope and WEBT Consortium, could give strong indications of the emission mechanisms involved in this source, in particular of the real nature of the soft X-ray excess, Seyfertlike features in the spectrum, and the possibility of the bulk Comptonization feature.

Finally, with two $\gamma$-ray satellites, AGILE and Fermi, in orbit at the same time, we will be able to study the source behavior at high energies in detail on a long timescale, even if a wide multiwavelength coverage is needed to achieve a complete understanding of the structure of the jet, the origin of the seed photons for the inverse Compton process, and all the emission mechanisms working in this blazar.

Acknowledgements. We thank the anonymous referee for the useful comments. The AGILE Mission is funded by the Italian Space Agency (ASI) with scientific participation by the Italian Institute of Astrophysics (INAF) and the Italian Institute of Nuclear Physics (INFN). We acknowledge the use of public data from the Swift data archive. We thank the Swift Team for making these observations possible, particularly the duty scientists and science planners. The Submillimeter Array is a joint project between the Smithsonian Astrophysical Observatory and the Academia Sinica Institute of Astronomy and Astrophysics and is funded by the Smithsonian Institution and the Academia Sinica. UMRAO is funded by a series of grants from the NSF and by the University of Michigan. The research has been supported by the Taiwan National Science Council grant No. 96-2811-M-008-058. This paper is partly based on observations carried out at the German-Spanish Calar Alto Observatory, which is jointly operated by the MPIA and the IAA-CSIC. Acquisition of the MAPCAT data is supported in part by the Spanish "Ministerio de Ciencia e Innovación" through grant AYA200767626-C03-03. Some of the authors acknowledge financial support by the Italian Space Agency through contract ASI-INAF I/088/06/0 for the Study of HighEnergy Astrophysics.

Facilities: AGILE, Swift, UMRAO and WEBT.

\section{References}

Abdo, A. A., Ackermann, M., Ajello, M., et al. 2009, ApJ, 700, 597

Arnaud, K. A. 1996, Astronomical Data Analysis Software and Systems V, ed. G. Jacoby, \& J. Barnes, ASP Conf. Ser., 101, 17

Bach, U., Raiteri, C. M., Villata, M., et al. 2007, A\&A, 464, 175

Barbiellini, G., Bordignon, G., Fedel, G., et al. 2001, Gamma 2001: Gamma-

Ray Astrophysics, ed. S. Ritz, N. Gehrels, \& C. R. Shrader, Amer. Inst. Phys. Conf. Ser., 587, 754

Barthelmy, S. D., Barbier, L. M., Cummings, J. R., et al. 2005, Space Sci. Rev., 120,143

Begelman, M. C., \& Sikora, M. 1987, ApJ, 322, 650

Bessell, M. S., Castelli, F., \& Plez, B. 1998, A\&A, 333, 231

Blandford, R. D., \& Rees, M. J. 1978, in BL Lac Objects, ed. A. M. Wolfe (Univ. Pittsburgh Press), 328

Bloom, S. D., \& Marscher, A. P. 1996, ApJ, 461, 657

Bolton, J. G., \& Ekers, J. 1966, Aust. J. Phys., 19, 559

Böttcher, M. 2007, Ap\&SS, 309, 95

Burbidge, E. M., \& Kinnan, T. D. 1966, ApJ, 145, 654

Burrows, D. N., Hill, J. E., Nousek, J. A., et al. 2005, Space Sci. Rev., 120, 165

Cardelli, J. A., Clayton, G. C., \& Mathis, J. S. 1989, ApJ, 345, 245

Cash, W. 1979, ApJ, 228, 939

Celotti, A., Ghisellini, G., \& Fabian, A. C. 2007, MNRAS, 375, 417

Ciprini, S., \& Corbel, F. 2009, ATel, 1897

Costa, E., Barbanera, L., Feroci, M., et al. 2001, X-ray Astronomy: Stellar Endpoints, AGN, and the Diffuse X-ray Background, ed. N. E. White, et al. (New York: AIP) 599, 582

Crummy, J., Fabian, A. C., Gallo, L., et al. 2006, MNRAS, 365, 1067

Cutini, S., \& Hays, E. 2009, ATel, 2033

D'Ammando, F., Bulgarelli, A., Vercellone, S., et al. 2008a, ATel, 1436

D’Ammando, F., Bianchi, S., Jiménez-Bailón, E., et al. 2008b, A\&A, 482, 499

D'Ammando, F., Vercellone, S., Tavani, M., et al., 2009, ATel, 1957

Dermer, C. D., Schlickeiser, R., \& Mastichiadis, A. 1992, A\&A, 256, L27

Donato, D., Ghisellini, G., Tagliaferri, G., et al. 2001, A\&A, 375, 739

Fabian, A. C. 1999, MNRAS, 308, L39

Feroci, M., Costa, E., Soffitta, P., et al. 2007, Nucl. Instr. Meth. Phys. Res. A, 581,728

Gambill, J. K., Sambruna, R. M., Chartas, G., et al. 2003, A\&A, 401, 505

Gehrels, N., Chincarini, G., Giommi, P., et al. 2004, ApJ, 611, 1005

Ghisellini, G., Maraschi, L., \& Tavecchio, F. 2009, MNRAS, 396, L105

Gierlinski, M., \& Done, C. 2004, MNRAS, 349, L7

Giuliani, A., Chen, A., Mereghetti, S., et al. 2004, Mem. Soc. Astron. Italiana Suppl., 5, 135

Giuliani, A., Cocco, V., Mereghetti, S., et al. 2006, Nucl. Instr. Meth. Phys. Res. A, 568, 692

Grandi, P., \& Palumbo, G. 2004, Science, 306, 998

Jorstad, S. G., Marscher, A. P., Lister, M. L., et al. 2005, AJ, 130, 1418

Hartman, R. C., Bertsch, D. L., Fichtel, C. E., et al. 1992, NASCP, 3137, 116

Hartman, R. C., Bertsch, D. L., Bloom, S. D., et al. 1999, ApJS, 123, 79

Kalberla, P. M. W., Button, W. B., Hartmann, D., et al. 2005, A\&A, 440, 775

Kataoka, J., Mattox, J. R., Quinn, J., et al. 1999, ApJ, 514, 138

Kataoka, J., Madejski, G., Sikora, M., et al. 2008, ApJ, 672, 787

Labanti, C., Marisaldi, M., Fuschino, F., et al. 2009, Nucl. Instr. Meth. Phys. Res. A, 598,470

Laor, 1990, MNRAS, 246, L369, 5

Lawson, A. J., \& Turner, M. J. L. 1997, MNRAS, 288, 920

Lister, M. L., Homan, D. C., Kadler, M., et al. 2009, ApJ, 696, L22

Malkan, M. A., \& Moore, R. L. 1986, ApJ, 300, 216

Maraschi, L., Ghisellini, G., \& Celotti, A. 1992, ApJ, 397, L5

Marscher, A. P., \& Gear, W. K. 1985, ApJ, 298, 114

Massaro, F., Tramacere, A., Cavaliere, A., et al. 2008, A\&A, 478, 395

Mattox, J. R. 1993, ApJ, 410, 609

Mattox, J. R., Bertsch, D. L., Chiang, J., et al. 1996, ApJ, 461, 396

Neugebauer, G., Oke, J. B., Becklin, E. E., et al. 1979, ApJ, 230, 79

Nieppola, E., Valtoja, E., Tornikoski, M., et al. 2008, A\&A, 488, 867

Perotti, F., Fiorini, M., Incorvaia, S., et al. 2006, Nucl. Instr. Meth. Phys. Res. A, 556,228

Pian, E., \& Treves, A. 1993, ApJ, 416, 130 
Pian, E. Urry, C. M., Maraschi, L., et al. 1999, ApJ, 521, 112

Poole, T. S., Breeveld, A. A., Page, M. J., et al. 2008, MNRAS, 383, 627

Prest, M., Barbiellini, G., Bordignon, G., et al. 2003, Nucl. Instr. Meth. Phys. Res. A, 501, 280

Pucella, G., Vittorini, V., D’Ammando, F., et al. 2008, A\&A, 491, L21

Pucella, G., D’Ammando, F., Tavani, M., et al., 2009, ATel, 1968

Raiteri, C. M., Villata, M., Lanteri, L., et al. 1998, A\&AS, 130, 495

Raiteri, C. M., Villata, M., Larionov, V. M., et al. 2007, A\&A, 473, 819

Raiteri, C. M., Villata, M., Larionov, V. M., et al. 2008, A\&A, 491, 755

Roming, P. W. A., Kennedy, T. E., Mason, K. O., et al. 2005, Space Sci. Rev., 120,95

Sambruna, R. M., Barr, P., Giommi, P., et al. 1994, ApJS, 95, 371

Sambruna, R. M., Tavecchio, F., Ghisellini, G., et al. 2007, ApJS, 669, 884

Siebert, J., Brinkmann, W., Drinkwater, M. J., et al. 1998, MNRAS, 301, 261

Sikora, M., Begelman, M. C., \& Rees, M. J. 1994, ApJ, 421, 153

Singh, K. P., Rao, A. R., \& Vahia, M. N. 1990, ApJ, 365, 455

Singh, K. P., Shrader, C. R., \& George, I. M. 1997, ApJ, 491, 515

Smith, P. S., Elston, R., \& Berriman, R. G. 1988, ApJ, 326, L39

Tanner, A. M., Bechtold, J., Walker, C. E., et al. 1996, AJ, 112, 62

Tavani, M., Barbiellini, G., Argan, A., et al. 2008, Nucl. Instr. Meth. Phys. Res. A, 588, 52

Tavani, M., Barbiellini, G., Argan, A., et al. 2009, A\&A, 502, 995

Tavecchio, F., \& Ghisellini, G. 2008, MNRAS, 386, 945980

Tavecchio, F., Maraschi, L., Ghisellini, G., et al. 2000, ApJ, 543, 535

Tavecchio, F., Maraschi, L., Ghisellini, G., et al. 2007, ApJ, 665, 980

Tramacere, A., Massaro, F., \& Cavaliere, A. 2007, A\&A, 463, 521

Tramacere, A. 2008, ATel, 1743

Türler, M., Chernyakova, M., Courvoisier, T. J.-L., et al. 2006, A\&A , 451, L1

Ulrich, M., Maraschi, L., \& Megan, C. M. 1997, ARA\&A, 35, 445

Urry, C. M., \& Padovani, P. 1995, Publ. Astron. Soc. Pac. J. Phys., 107, 803

Vercellone, S., Chen, A. W., Giuliani, A., et al. 2008, ApJ, 676, L13

Vercellone, S., D’Ammando, F., Pucella, G., et al. 2009, ATel, 1976

Villata, M., Raiteri, C. M., Aller, M. F., et al. 2007, A\&A, 464, L5

Villata, M., Raiteri, C. M., Larionov, V. M., et al. 2008, A\&A, 481, L79

Wardle, J. F. C., Homan, D. C., Cheung, C. C., et al. 2005, ASPC, 340, 67

Wills, B. J, Netzer, H., \& Wills, D. 1985, ApJ, 288, 94

Woo, J., \& Urry, C. M. 2002, ApJ, 579, 530

1 INAF/IASF - Roma, via Fosso del Cavaliere 100, 00133 Roma, Italy

e-mail: filippo.dammando@iasf-roma.inaf.it

2 Dip. di Fisica, Univ. di Roma "Tor Vergata", via della Ricerca Scientifica 1, 00133 Roma, Italy

3 INAF, Oss. Astronomico di Torino, via Osservatorio 20, 10025 Pino Torinese (Torino), Italy

${ }^{4}$ CIFS - Torino, viale Settimio Severo 3, 10133 Torino, Italy

5 INAF, Istituto di Astrofisica Spaziale e Fisica Cosmica, via U. La Malfa 153, 90146 Palermo, Italy

${ }^{6}$ Dip. di Fisica and INFN Trieste, via Valerio 2, 34127 Trieste, Italy

7 INFN - Pavia, via Bassi 6, 27100 Pavia, Italy

${ }^{8}$ Dip. di Fisica Nucleare e Teorica, Univ. di Pavia, via Bassi 6, 27100 Pavia, Italy

9 INAF/IASF - Bologna, via Gobetti 101, 40129 Bologna, Italy

10 INAF/IASF - Milano, via E. Bassini 15, 20133 Milano, Italy
11 Dip. di Fisica Generale dell’Universitá, via P. Giuria 1, 10125 Torino, Italy

12 ENEA - Bologna, via dei Martiri di Monte Sole 4, 40129 Bologna, Italy

13 INFN - Roma "La Sapienza", Piazzale A. Moro 2, 00185 Roma, Italy

14 INFN - Roma "Tor Vergata", via della Ricerca Scientifica 1, 00133 Roma, Italy

15 INAF - Oss. Astronomico di Cagliari, loc. Poggio dei Pini, strada 54, 09012 Capoterra (CA), Italy

16 Dip. di Fisica, Univ. dell'Insubria, via Valleggio 11, 22100 Como, Italy

17 ENEA - Roma, via E. Fermi 45, 00044 Frascati (Roma), Italy

18 Instituto de Astrofísica de Andalucía, CSIC, Apartado 3004, 18080 Granada, Spain

19 Department of Astronomy, University of Michigan, Ann Arbor, MI 48109, USA

20 Pulkovo Observatory, Russian Academy of Sciences, 196140 St.-Petersburg, Russia

21 MPIfR, 53121 Bonn, Germany

22 Instituto de Astronomia, Universidad Nacional Autonoma de Mexico, Mexico, D. F. Mexico

23 Tuorla Observatory, Department of Physics and Astronomy, University of Turku, Väisäläntie 20, 21500 Piikkiö, Finland

24 Astron. Inst., St.-Petersburg State Univ., 198504 St.-Petersburg, Russia

25 INAF - Osservatorio Astrofisico di Catania, via S. Sofia 78, 95123 Catania, Italy

${ }^{26}$ Institute of Astronomy, National Central University, Taiwan

${ }^{27}$ Lulin Observatory, Institute of Astronomy, National Central University, Taiwan

28 INAF, Osservatorio Astronomico di Roma, via di Frascati 33, 00040, Monte Porzio Catone, Italy

29 INAF, Osservatorio Astronomico di Collurania, via Mentore Maggini, 64100 Teramo, Italy

30 Harvard-Smithsonian Center for Astrophysics, Cambridge, Garden st. 60, MA 02138, USA

31 Institute for Astrophysical Research, Boston University, 725 Commonwealth Avenue, Boston, MA 02215, USA

32 ZAH, Landessternwarte Heidelberg, Königstuhl, 69117 Heidelberg, Germany

33 Instituto de Astronomia, Universidad Nacional Autonoma de Mexico, 2280 Ensenada, B.C. Mexico

34 Abastumani Observatory, 383762 Abastumani, Georgia

35 INAF - IRA, contrada Renna Bassa, 96017 Noto (SR), Italy

36 Department of Physics and Astronomy, University of Southampton S17 1BJ, UK

37 INAOE, Apdo. Postal 51 y 216, 72000 Tonantzintla, Puebla, Mexico

38 Lowell Observatory, Flagstaff, AZ 86001, USA

39 ASI-ASDC, via G. Galilei, 00044 Frascati (Roma), Italy

40 ASI, viale Liegi 26, 00198 Roma, Italy 